\title{
EDITORIALS
}

\section{Turning and Churning: Loss of Health Insurance Among Adults in Medicaid}

\author{
Milda R. Saunders, MD, MPH and G. Caleb Alexander, MD, $M S^{2,3,4,5}$ \\ ${ }^{1}$ Section of Hospital Medicine, Department of Medicine, University of Chicago Hospitals, Chicago, IL, USA; ${ }^{2}$ Section of General Internal \\ Medicine, Department of Medicine, University of Chicago Hospitals, Chicago, IL, USA; ${ }^{3}$ MacLean Center for Clinical Medical Ethics, University \\ of Chicago, Chicago, IL, USA; ${ }^{4}$ Center for Health and the Social Sciences, University of Chicago, Chicago, IL, USA; ${ }^{5}$ Department of Pharmacy \\ Practice, University of Illinois at Chicago School of Pharmacy, Chicago, IL, USA.
}

$\mathrm{J}$ Gen Intern Med 24(1):133-4

DOI: $10.1007 / \mathrm{s} 11606-008-0861-0$

(c) Society of General Internal Medicine 2008

$\mathrm{H}$ aving health insurance is good, but keeping it over time is even better. Despite this, millions of Americans experience gaps in their health care coverage. This is a particular concern for non-elderly adults covered by Medicaid, who most commonly lose eligibility due to increases in income or loss of categorical eligibility that they have through receipt of Social Security Income (SSI) or Temporary Assistance to Needy Families (TANF). ${ }^{1}$ Of course, Medicaid eligibility and enrollment are not synonymous, and even among those with continuous eligibility, drop out may still occur, often due to the administrative requirements of reenrollment.

Substantial evidence exists regarding the potential harmful impact that loss of Medicaid insurance, whether through lost eligibility or drop out, can have. In many cases when someone loses Medicaid coverage, the insurance is not immediately replaced by an alternative, thus rendering a large population, at least temporarily, uninsured ${ }^{2}$. Even after accounting for potentially confounding factors, loss in coverage is associated with discontinuity of care ${ }^{3-5}$, reduced ambulatory care use $e^{6}$, increased emergency room use, ${ }^{7,8}$ higher health care costs, ${ }^{7}$ and worse patient outcomes. ${ }^{9}$ Furthermore, children of adults who lose Medicaid may themselves be affected through "spillover effects" and are more likely to be uninsured despite their eligibility for coverage. ${ }^{10,11}$ While many studies examining the association between health insurance and outcomes of care are plagued by unwarranted casual inference, the strength of evidence supports the importance of insurance in improving specific health outcomes, especially for specific vulnerable populations such as those eligible for Medicaid.

In this issue of JGIM, Sommers ${ }^{12}$ adds to our knowledge of Medicaid disenrollment among both non-elderly adults and children. ${ }^{2,13-15}$ This analysis is important because of considerable federal changes due to welfare reform and citizenship documentation and state changes in Medicaid eligibility during the past decade. Using data from the 2000-2004 Medical Expenditure Panel Survey (MEPS), Sommers examines annual disenrollment by identifying insurance coverage after 12 months for individuals enrolled in Medicaid at survey outset. He also provides a survival analysis of newly enrolled individuals, which provides information on lengths of typical Medicaid enrollment

Published online December 19, 2008 as well as Medicaid churning, disenrollment with re-enrollment at a later date. Among all adults enrolled in Medicaid, 21\% were no longer enrolled 12 months later, and 14\% were uninsured. Survival analysis of newly enrolled individuals showed that $20 \%, 43 \%$ and $55 \%$ of adults had disenrolled within 6,12 , 23 months from their initial enrollment in Medicaid. Children were less likely than adults to be disenrolled, with rates of $12 \%$, $26 \%, 36 \%$ at 6,12 , and 23 months, respectively.

This report complements other studies of Medicaid disenrollment by providing an update as well as attempting to infer whether observed rates of disenrollment are due to loss of categorical eligibility, loss of need (i.e. increased income or acquisition of other insurance), or loss of coverage despite continued eligibility. A survival analysis examining "always eligible" adults-low-income pregnant women and those receiving SSI and TANF-identifies significant levels of drop out even among this group, though rates were considerably lower than among all adults. A complementary survival analysis stratified by those whose income increased $(>\$ 10,000)$ or decreased/stayed nearly the same demonstrated no significant difference in disenrollment between the two groups, once again suggesting that disenrollment was not primarily driven by eligibility-related factors. Although rates of Medicaid loss were modestly greater among some demographic groups, interestingly, there were no differences in the likelihood of subsequent lack of insurance based on individuals' self-reported health or chronic conditions. This is important, given that the benefits of insurance coverage may be considerably greater among those with poor self-reported health and greater comorbid illness than among their counterparts.

Despite Sommers' effort to identify the mechanisms that account for disenrollment, important questions remain. Perhaps the greatest limitation of the current analysis is the inability to address state-level differences in Medicaid design. Evaluating these differences is crucial both to identify mechanisms that account for Medicaid turnover as well as to evaluate the outcomes of this phenomenon. Given that Medicaid programs are developed, administered, and financed by individual states, in some sense "Medicaid" represents 50 different programs, all with the same name. Analyses that exploit the rich state-to-state variation in Medicaid design, whether through the use of restricted MEPS files or other sources, allow for an examination of state specific rates of disenrollment within diverse policy contexts. ${ }^{16}$ These analyses can be quite informative, since states vary remarkably with regards to their populations, as well as characteristics of their Medicaid programs, including eligibility requirements, enrollment criteria, and allocation of resources across different Medicaid services. 
States face several challenging issues regarding their Medicaid enrollment policies. In most states, the health care safety net is a hodge-podge of programs that provide coverage to poor children and families. Due to complex eligibility rules, many states rely on outdated and unwieldy automated systems to determine eligibility. ${ }^{17}$ Additionally, byzantine program requirements may impose an administrative burden on individuals who are least able to navigate the system due to low literacy, limited English-language proficiency and, for some, chaotic daily lives. Failure to complete or return paperwork or inadequate supporting documents is responsible for lapses in coverage for many families. ${ }^{17,18}$

Of course, matters of political expediency may also lead states to resist efforts to ease the process of Medicaid (re)enrollment. Administrative barriers may have the effect-intended or not-of reducing the ranks of the enrolled, which may be helpful for states struggling to balance their budgets in a second-best world. However, there is at least some evidence that such practice may be penny-wise, pound-foolish, since gaps may shift, rather than decrease, costs. For example, a recent study estimated that a 10\% loss in Medicaid coverage among children in the Phoenix area would increase the costs of health care in the community by $\$ 3.5$ million annually, or $\$ 2,121$ for each disenrolled child. ${ }^{7}$ This cost increase was attributed to a shift of care from ambulatory settings to more expensive emergency departments and an increase in hospital stays. In some cases, states may still end up picking up these costs, in the form of reimbursement for uncompensated care through the emergency department or costs at public hospitals and clinics. However, in other settings, costs may be shifted not only downstream, but to other payers. ${ }^{19}$ For example, hospitals may pass on costs of uncompensated care to privately insured patients and other payers. Counties may help absorb the costs through countybased hospitals and public health clinics.

So where do we go from here? Sommers' work underscores the importance of focusing research and reform efforts not only on making Medicaid policies, but implementing them into practice. His analysis serves as an important reminder that a considerable portion of Medicaid disenrollment for non-elderly adults continues to be due to administrative barriers rather than lost eligibility. Given political will, relatively simple changes may reduce these rates of disenrollment. At the federal level, linkage of state reimbursement to the proportion of Medicaid eligibles that are actually Medicaid-covered would increase states' incentives to simplify the (re)enrollment process. Some states, upon seeing how their disenrollment rate compares to other states and how much this may end up costing them (in lost federal matching, lost wages, and paying for uncompensated care) might reduce administrative barriers to (re)enrollment. Individual states could also take other steps, such as reducing the requirement for in-person interviews for renewals, allowing for continuous 12-month eligibility rather than more frequent re-enrollment, helping Medicaid recipients better manage the renewal process either through case management ${ }^{20}$ or extensions in Medicaid managed care, and automatically (re)enrolling those categorically eligible through their SSI or TANF program office.

The United States now has a new president who is inheriting a host of challenges and opportunities regarding health care reform. During the presidential election, there was considerable discussion of methods of increasing coverage the 47 million Americans who are currently uninsured and making private or employer-sponsored insurance more affordable. ${ }^{21}$ However, neither candidates' health plans offered much consideration regarding the current states' Medicaid programs, and the role that Medicaid can play in providing greater coverage for the nearly 60 million poor and disabled Americans. Given the findings of Sommers and others' reports, clearly there is more work to do.

Corresponding Author: Milda R. Saunders, MD, MPH; The University of Chicago, 5841 S. Maryland, MC 2007, Chicago, IL 60637, USA (e-mail: msaunder@medicine.bsd.uchicago.edu).

\section{REFERENCES}

1. Medicaid Program-General Information: Technical Summary. 12/14/05; (Accessed October 31. 2008 at http://www.cms.hhs.gov/MedicaidGenInfo/ 03_TechnicalSummary.asp\#TopOfPage.

2. Klein K, Glied S, Ferry D. Entrances and exits: health insurance churning, 1998-2000: Commonwealth Fund; 2005.

3. Fairbrother G, Jain A, Park HL, et al. Churning in Medicaid managed care and its effect on accountability. J Health Care Poor Underserved. 2004; 15(1):30-41.

4. Fairbrother GL, Emerson HP, Partridge L. How stable is Medicaid coverage for children? Health Aff. 2007;26(2):520-8.

5. Weissman JS, Witzburg R, Linov P, Campbell EG. Termination from Medicaid: how does it affect access, continuity of care, and willingness to purchase insurance? J Health Care Poor Underserved. 1999; 10(1):122-37.

6. Carlson MJ, DeVoe J, Wright BJ. Short-term impacts of coverage loss in a Medicaid population: early results from a prospective cohort study of the Oregon Health Plan. Ann Fam Med. 2006;4(5):391-8.

7. Rimsza ME, Butler RJ, Johnson WG. Impact of Medicaid disenrollment on health care use and cost. Pediatrics. 2007;119(5):e1026-32.

8. Lowe R, McConnell K, Vogt M, Smith J. The impact of Medicaid cutbacks on emergency department use: the Oregon experience. Annals of Emergency Medicine. 2008.

9. Weissman JS, Stern R, Fielding SL, Epstein AM. Delayed access to health care: risk factors, reasons, and consequences. Ann Intern Med. 1991;114(4):325-31.

10. Sommers BD. Insuring children or insuring families: do parental and sibling coverage lead to improved retention of children in Medicaid and CHIP? J Health Econ. 2006;25(6):1154-69.

11. DeVoe JE, Krois L, Edlund T, Smith J, Carlson NE. Uninsurance among children whose parents are losing Medicaid coverage: Results from a statewide survey of Oregon families. Health Serv Res. 2008;43(1 Pt 2):401-18.

12. Sommers BD. Loss of Health Insurance Among Non-elderly Adults in Medicaid. J Gen Intern Med. XXXXXX, 2008

13. Fairbrother G, Jain A, Park HL, et al. Churning in Medicaid managed care and its effect on accountability. J Health Care Poor Underserved. $2004 ; 15(1): 30-41$.

14. Fairbrother GL, Emerson HP, Partridge L. How stable is Medicaid coverage for children. Health Aff (Millwood). 2007;26(2):520-8.

15. Carrasquillo O, Himmelstein DU, Woolhandler S, Bor DH. Can Medicaid managed care provide continuity of care to new Medicaid enrollees? An analysis of tenure on Medicaid. Am J Public Health. 1998;88(3):464-6

16. Weissman JS, Zaslavsky AM, Wolf RE, Ayanian JZ. State Medicaid coverage and access to care for low-income adults. J Health Care Poor Underserved. 2008;19(1):307-19.

17. Ellwood M. The Medicaid eligibility maze: coverage expands but problems persist-findings from a five stage study. Washington, DC: The Urban Institute; 1999.

18. Summer L, Mann C. Instability of public insurance coverage for children and their families: causes, consequences and remedies. New York, NY: The Commonwealth Fund: 2006.

19. Hadley J, Holahan J, Coughlin T, Miller D. Covering the uninsured in 2008: current costs, sources of payment and incremental costs. Health Aff (Millwood). 2008;27(5):399-415.

20. Fairbrother G, Stuber J, Dutton M, Scheinmann R, Cooper R. An examination of enrollment of children in public health insurance in New York City through facilitated enrollment. J Urban Health. 2004;81(2):191-205.

21. The uninsured: a primer. Key facts about Americans without insurance. Washington, DC: Kaiser Family Foundation: 2008, October. 